\title{
HORMESIS, SCREENING AND PRACTICAL USE OF BIOSTIMULATORS IN AGRICULTURE
}

\author{
Dascaliuc Al. \\ Institute of Genetics, Physiology and Plant Protection, st. Paduriy 20, Chisinau, 2002-MD, \\ Republic of Moldova, E-mail: dascaliuca@yahoo.com
}

\begin{abstract}
The term hormesis describes the biphasic response of any biological system to increasing the dose of the stress factor of a different nature, characterized in that low doses have stimulating, beneficial effects. In contrast, high doses cause harmful, inhibitory effects. The hormonal response is practically universal, being stimulated by the action of toxic substances, heavy metal ions, hormones, including physical factors. The standard type of response to different factors suggests installing these evolving mechanisms, so they are of particular interest in elucidating plant adaptation mechanisms to various stressors, including developing screening methods and practical use of biostimulators. The practical use of hormesis principles was the theoretical basis for elaboration and rational use of the biostimulator Reglalg in agriculture.
\end{abstract}

Keywords: adaptation, hormesis, stress, biostimulators.

In ontogenesis, plants face several challenges caused by abiotic factors such as extreme temperatures and biotic ones such as viral infections. During evolution, biological systems that respond effectively to environmental disturbances have survived, due to adaptations changing the internal environment and reaching stable homeostasis [1]. The impetuous development of science and technology has ensured the rapid and economically advantageous cultivation and protection of agricultural plants. In such conditions are mainly considered an economic advantage. Because of this, the long-term effects of new technologies, which led to their bankruptcy, have been ignored. The current reintroduction of organic farming and the widespread use of biostimulators of natural origin [1] respond to this situation. This transition can be rational if the systemic analysis of the factors that determine the resistance and productivity of organisms in specific environmental conditions applied. In this paper, we tend to briefly analyze the general principles that determine the effectiveness of screening the biostimulators and agriculture application.

Higher plants adapt at the molecular, cellular, and integral level to specific, often critical environmental conditions during ontogenesis. Increasing the efficiency of agricultural practice due to the involvement of various new technological processes has influenced both the cultivation environment (which also includes different biological systems) and the physiological state of cultivated plants. Under changing environmental conditions, plant survival depends on their resistance and ability to recover the damage caused by stress. Thus, plants' ability to tolerate different stress factor doses is the essential criterion determining their resistance. Research with various biological systems has shown that the response to stress factor doses is biphasic: after low doses, the answer is adaptive and beneficial, but high doses are harmful to plants. This response has been called hormesis, defined as "a process in which exposure to a low dose of a chemical agent or environmental factor that is damaging at higher doses induces an adaptive beneficial effect on the cell or organism" [2]. Hormetic dose-response curves manifest within a range of sub-inhibitory doses that are stimulant, with a peak at the maximum stimulation dose and end at the no observable adverse level, which typically precedes the inhibitory doses [3].

The biphasic type of response is characteristic of all biological systems. The establishment of the molecular mechanisms of plants' adaptive response to different "stressors" occurred throughout the evolutionary period. Thus, due to specific evolutionary 
pathways, the doses of physical and chemical factors that cause stress in one species' representatives become optimal for other species. An adaptive, positive effect in different species appears at different doses. It manifests itself in changes in the organism's phenotype and properties in response to changes in the environment. Consequently, hormesis provides a quantitative assessment of an organism's biological plasticity rather than otherwise reflection of biological systems' homeodynamic nature, rather than homeostasis (a process that maintains the stability of biosystems' internal environment changes in external conditions).

As a result of analyzing the concept of homeostasis and its relationship with other fundamental biological concepts, it is easy to conclude the relations between this phenomenon and many other adaptation phenomena. The analysis of these relationships can open up new perspectives in explaining and optimizing growth regulators' practical application to increase plant resistance to stress factors. Optimal concentrations (doses) of growth regulators are at a low level. The limits of stimulating doses usually occupy one, rarely two orders of magnitude, the zone of hormesis reaction are specific for various plant species. In high concentrations, they have an inhibitory, damaging effect on plants. Similarly, hormesis manifests itself after exposure to low doses of factors on the body; the hormesis zone's dosing interval rarely reaches two orders of magnitude. At high doses, it has a destructive effect on the body. Also, the expansion of the homeodynamic space during hormesis is in good harmony with the same effects of low doses of growth regulators. Moreover, the quantitative values of the positive impact of hormesis and growth regulators on plants' viability and productivity are comparable. Under optimal conditions, these effects reach $30 \%$ and quite rarely $-50 \%$. The positive impact of doses of stress-causing hormesis or optimal concentrations of growth regulators, on many endogenous processes and cellular signaling systems, on which cell division depends, the growth and productivity of plants are related. We assume that the common elements that characterize hormesis and the effects of growth regulators are not random. In both cases, the homeodynamic equilibrium of the biosystems expands. Thus, the analysis of the concepts of biological phenomena during hormesis and the action of growth regulators can ensure the rapid development of using biostimulants' theory and practice.

During the development of the biostimulator Reglalg and the optimization of its practical use conditions, we considered the complexity of the biostimulator chemical content based on the hormesis. The phenomenon of hormesis, manifesting it after applying different chemical and physical components, is universal for organisms with varying organization levels. Thus, the preparation application can cause specific effects on the plant, pests, and interactions between the plant and pests. The biostimulator Reglalg contains several secondary metabolites and other active components. Due to its complexity, it simultaneously influences the morphogenesis, ontogenesis, and adaptive capacity of plants. In excess, they may have harmful effects. Multiannual research has shown that Reglalg has eliciting effects, inducing plant tolerance to abiotic and biotic stresses. At the same time, it influences the morphogenesis of plants, contributing to the formation of characteristics that involve the phenomena of avoiding the action of biotic and abiotic stress factors.

Reglalg is a preparation containing a mixture of microelements, unsaturated fatty acids, aldehydes, ketones, aldehyde-ketones, and other bioactive components, extracted from algae in particular conditions. The successful combination of such ingredients presupposes a broad spectrum of Reglalg activity. The Reglalg stimulates the development of the root system, increases (conditioning) ability of plants to form systemically acquired resistance, increases their ability to repair damage caused by stressors. The characteristic active ingredients for Reglalg are dissolved in $20^{\circ}$ ethyl alcohol, convenient for storage and use. Before spraying, one liter of the Reglalg must be is dissolved in 100-300 liters (for seeds) or 400- 
1000 liters (for plants) of water, or solutions of protective substances. In cereals, the biological effectiveness of the preparation consists of reducing the hypocotyl's length, stimulating the root system's development, and increasing its ability to recover from stress. Due to the complex and harmonious composition of active principles, Reglalg stimulates vigor, maintains and restores homeostasis, and increases plants' resistance to stress factors. Promoting the development of the root system, reducing the length of the epicotyl, and deepening the growth cone's position in the soil, the plants treated with the Reglalg are avoiding (diminishing) the doses stress factors (drought, heat, frost). The preparation contributes to the symbiosis interactions between the soybean plants and the soil organisms. Reglalg exerts triple action on plants: increases the vigor (1), resistance (2), and recovery (3) of stress-related damage. In this way, it exhibits several beneficial effects on plants;

1. Contributes to avoiding (diminishing) the action of stressors;

2. Enables the reduction of doses of protection preparations and stimulates the interactions between the plant and beneficial organisms in the soil;

3. In the complex, these effects ensure the increase of the crop's quantity and quality by stimulating the natural processes while also reducing the environment's pollution by synthetic protective substances. Substances that are part of Reglalg influenced the growth and development of plants in different ways. The multitude of induced processes ensures the extension of conditions for maintaining homeostasis and accelerating restoring homeostasis processes disturbed by stress. The biostimulator Reglalg accelerates the recovery processes from the stress factor's damage along with increasing plant resistance. It has systemic activity, retains beneficial activity in a wide range of concentrations and working environment. Due to this, the doses of biostimulator use are flexible. Contributing the increase of viability and capacity of plants to maintain, or restore, homeostasis under stress, Reglalg induces enzymatic and metabolic systems to neutralize the reactive oxygen species and intensify the recovery of stress-related damages. It acts as a biostimulator that stimulates roots growth in specific concentrations, activates secondary roots formation and development, and provides better mineral and water supply to plants.

Initial relative delay of shoots growth of plants obtained from the seeds treated with Reglalg is accompanied by the stimulation of their growth and development during the next periods of vegetation. On cereals, the use of Reglalg ensures a shortening of the length of hypocotyl and, consequently, the deeper installation of the tillering node in the soil. This morphological change contributes to decreasing winter frost's deteriorative effects, high temperatures, and drought during summer. Under these conditions, it increases the number of productive plants, increases the photosynthetic activity, and prolongs the plants' vegetation duration by about one week.

Reglalg components increase plant resistance to frost, drought, high temperature, and snow mold. In the spring, plants with a well-developed roots system are more viable, resistant to disease (snow mold). Thanks to this, plants use the soil nutrients and solar energy more efficiently, which is manifested in increasing the chlorophyll content and photosynthesis effectiveness, especially during the grain maturation period, thus contributing to increased carbon dioxide binding and sugar biosynthesis. The stimulating effects of Reglalg on plant productivity are a consequence of the induction of avoidance phenomena (reduction of effective doses of stresses) and the increase of plant resistance to stress factors. When plants are treated with Reglalg in a mixture with fungicides and pesticides during the growing season, it enhances their activity and facilitates the reduction of plant protectors' effective doses. In soybean, seed treatment with Reglalg, besides promoting plant vigor, ensures the stimulation of symbiosis by stimulating the number of nodules and their mass. These occur 
even when the use of herbicides inhibits the effects of symbiosis. Under the influence of Reglalg, which is used concomitantly with the substances for the protection by spraying grapevine and fruit plants three times during the growing season, increases the annual growth, vigor, and productivity of plants, the beneficial effects also manifested during the next year. The Reglalg canlbe recommended treating seed before sowing and plants during the spring period. The results of infection with fungi and microbes do not occur or are very weak. The Reglalg canlbe recommended treating seed before sowing and plants during the spring period. The results of infection with fungi and microbes do not occur or are very weak.

The plant's response to the preparation action is similar to the hormonal responses to toxins and phytohormones. Thus, by managing the hormonal reactions in plants using biostimulators, meaningful results can be obtained to increase food production quality while minimizing their adverse effects. The action of the biostimulator in small doses can also stimulate the activity of fungi, bacteria, and other harmful organisms. These effects can occur by stimulating spores' growth, mycelium development, toxins production, etc. These stimulating effects can occur in production conditions, orchards, and nurseries after applying inappropriate doses of biostimulators and fungicides to reduce costs for protection. Exposure to sub-inhibitory doses may accidentally appear in agricultural fields, orchards, nurseries, and greenhouses, in various circumstances, such as improper application of the fungicide, lowdose applications to reduce costs for protection. These hormetic effects can be extremely harmful, reducing the quantity and quality of production. Unfortunately, at present, the impact of stimulating the development of plant pathogens, induced by herbicides, are poorly understood. Thus it is difficult to exclude the mentioned unexpected effects when the design of chemical management strategies. There is a need to promote well-argued practices for the responsible management and use of biostimulators and safeners. Hormesis is a relatively new concept. The use of this concept in agriculture for disease management can open up new opportunities to improve crops' quality and productivity, with the main focus being on innate plant immunity, which is one of the most important mechanisms for adapting to the environment.

\section{Conclusions}

To survive, plants have to respond to stressors during the life cycle. After being exposed to doses of stress factors below a certain level, they withstand the exposure to higher levels of stress factors due to hormetic response to stress. This reaction can increase after action with different physical and chemical components, including biostimulators. Knowledge of the hormonal mechanisms makes it possible to optimize the methods of using biostimulators, reducing the doses and the frequency of application of protective substances in agriculture.

\section{References}

1. Dascaliuc A., Ivanova R., Arpentin Gh. Systemic approach in determining the role of bioactive compounds." In Pierce G.N.; Mizin VI; Omelchenko eds. Advanced bioactive compounds countering the effects of radiological, chemical, and biological agents, Strategies to counter biological damage; Series: NATO Science for Peace and Security Series A: Chemistry and Biology. Springer, 2013. p. 121-131.

2. Calabrese E.J., Bachmann K. A., Bailer A J. et al. Biological stress response terminology: Integrating the concepts of adaptive response and preconditioning stress within a hormetic dose-response framework// Toxicol. Appl. Pharmacol., 2007, v. 222, n. 1, p. 122-128.

3. Mattson M.P, Calabrese E.J. Hormesis a revolution in biology, toxicology and medicine. Springer New York Dordrecht Heidelberg London, 214. 